\title{
Certainty equivalence implies detectability ${ }^{1}$
}

\author{
J.P. Hespanha ${ }^{\mathrm{a}, *}$, A.S. Morse ${ }^{\mathrm{b}}$ \\ ${ }^{a}$ Electronics Research Laboratory, University of California, Berkeley, CA 94720, USA \\ ${ }^{\mathrm{b}}$ Center for Computational Vision and Control, Yale University, New Haven, CT 06520, USA
}

Received 2 October 1997; received in revised form 10 June 1998; accepted 12 June 1998

\begin{abstract}
It is shown that any stabilizing, certainty equivalence control used within an adaptive control system, causes the familiar interconnection of a controlled process and associated output estimator to be detectable through the estimator's output error $e_{p}$, for every frozen value of the index or parameter vector $p$ upon which both the estimator and controller dynamics depend. The fact that certainty equivalence implies detectability has been known for some time - this has been shown to be so whenever the process model is linear and the controller and estimator models are also linear for every frozen value of $p$. In this paper, use is made of recently introduced concepts of input-to-state stability and detectability for nonlinear systems to prove that the same implication is valid in a more general, nonlinear setting. (C) 1999 Published by Elsevier Science B.V. All rights reserved.
\end{abstract}

Keywords: Certainty equivalence; Detectability; Input-to-state stability; Adaptive control

\section{Introduction}

"Certainty equivalence ${ }^{2}$ " is a well-known heuristic idea which advocates that in an adaptive context, the feedback control to an imprecisely modeled process should, at each instant of time, be designed on the basis of a current estimate of what the process model is, with the understanding that each such estimate is to be viewed as correct even though it may not be. On the surface justification for certainty equivalence seems self-evident: if process model estimates converge to the "true" process model, then a certainty equivalence based controller ought to converge to the nonadaptive controller which would have been

\footnotetext{
*Corresponding author. E-mail: hespanha@robotics.eecs. berkeley.edu.

${ }^{1}$ This research was supported by the Air Force Office of Scientific Research, the Army Research Office, and the National Science Foundation.

${ }^{2}$ For a historical account of this term's origin, see [4].
}

implemented had there been no process uncertainty. The problem with this justification is that because of noise and unmodeled dynamics, process model estimates do not typically converge to the true process model - even in those instances where certainty equivalence controls can be shown to perform in a satisfactory manner. A more plausible justification stems from the fact that any \{stabilizing certainty equivalence control causes the familiar interconnection of a controlled process and associated output estimator to be detectable through the estimator's output error $e_{p}$, for every frozen value of the index or parameter vector $p$ upon which both the estimator and controller dynamics depend. Detectability is key because adaptive controller tuning/switching algorithms are invariably designed to make $e_{p}$ small - and so with detectability, smallness of $e_{p}$ ensures smallness of the state of the controlled process and estimator interconnection. The fact that certainty equivalence implies detectability has been known for some time - this has been 
shown to be so whenever the process model is linear and the controller and estimator models are also linear for every frozen value of $p[10,12]$. In this paper use is made of recently introduced concepts of input-tostate stability [2] and detectability $[8,14]$ for nonlinear systems, to explain why the same implication is valid in a more general, nonlinear setting.

The overall problem of interest is to regulate about its zero state, the state of a very imprecisely modeled process (Section 2). The process is assumed to admit a dynamical model which is in the union of a number of subclasses, each subclass being small enough so that each of its members can be adequately regulated with a single fixed dynamical controller. Such a family of controllers is taken as given. The idea then is to switch various members of this family into feedback with the process (Section 3 ). Switching is carried out from time to time by an estimator-based supervisor (Section 5) which bases controller selection on certainty equivalence. The concepts of input-tostate stability and detectability are briefly discussed in Section 4 and are then used in Section 6 to explain the implication of using a certainty equivalence strategy. The notion of an "injected system" is made precise in Section 7 and the concept of "state sharing" is explained in Section 8. Finally in Section 9, the utility of this paper's main result, namely the observation that certainty equivalence implies detectability, is demonstrated by proving that in a fairly general setting, the supervisory control architecture we have described, solves the overall problem formulated in Section 2.

Notation. In the sequel, prime denotes transpose and $\|x\|$ denotes the 2-norm of a vector $x$ in a real, finitedimensional space $\mathscr{X}$. The exterior direct sum of two real linear spaces $\mathscr{X}$ and $\mathscr{Y}$, is written as $\mathscr{X} \oplus \mathscr{Y}$. We write $\mathscr{L}^{1}\left[t_{1}, t_{2}\right)$ for the space of all real, vector-valued functions $f$ on $\left[t_{1}, t_{2}\right)$ for which $\int_{t_{1}}^{t_{2}}\|f(\tau)\| \mathrm{d} \tau<\infty$.

\section{Overall problem}

Let $\mathbb{P}$ denote the model of a process with a control input $u$, a measured output $y$, and a piecewisecontinuous disturbance/noise input $w$ that cannot be measured. Suppose that $u, y$, and $w$ take values in real, finite-dimensional vector spaces $\mathscr{U}, \mathscr{Y}$, and $\mathscr{W}$, respectively. Assume that $\mathbb{P}$ is an unknown member of some suitably defined family of dynamical systems $\mathscr{F}$ whose elements each have a real, finite-dimensional state space $\mathscr{X}_{\mathbb{P}}$ and a pair of defining equations of the form

$\dot{x}_{\mathbb{P}}=A_{\mathbb{P}}\left(x_{\mathbb{P}}, w, u\right), \quad y=C_{\mathbb{P}}\left(x_{\mathbb{P}}, w\right)$,

where $A_{\mathbb{P}}$ and $C_{\mathbb{P}}$ are at least locally Lipschitz continuous on $\mathscr{X}_{\mathbb{P}} \oplus \mathscr{W} \oplus \mathscr{U}$ and $\mathscr{X}_{\mathbb{P}} \oplus \mathscr{W}$, respectively. Assume, in addition, that $\mathscr{F}$ can be written as

$\mathscr{F}=\bigcup_{p \in \mathscr{P}} \mathscr{F}_{p}$,

where $\mathscr{P}$ is either a finite set of indices or a compact subset of parameter values within a real, finitedimensional, normed linear space. Here each $\mathscr{F}_{p}$ denotes a subfamily consisting of a given nominal process model $\mathbb{M}_{p}$ together with a collection of "perturbed versions" of $\mathbb{M}_{p}$. Typically one would require the allowable perturbations to be "small" enough so that for each possible process model $\mathbb{P}$ in $\mathscr{F}$, there is a nominal process model within the set $\mathscr{M} \triangleq\left\{\mathbb{M}_{p}: p \in \mathscr{P}\right\}$, which is "close" to $\mathbb{P}$ in some suitably defined sense. We shall not explicitly demand that this be so, since the main result of this paper does not require it.

The overall problem of interest is to devise a feedback control which regulates $y$ about the value 0 and, in addition, causes $y$ to tend to 0 in the event that $w$ tends to zero. The main result of this paper, Theorem 1 , applies to this problem and \{with minor modification\} to more general tracking problems such as set-point control where the references to be tracked are generated by exogenous input-free, time-invariant dynamical systems. In the event that the class within which $\mathbb{P}$ resides - say $\mathscr{F}_{p^{*}}$ - were known and fixed, the problem might be dealt with using standard nonadaptive techniques. On the other hand, if $\mathbb{P}$ were to change slowly or intermittently from time to time, and the corresponding value of $p^{*}$ over time were known or could be determined from measured data, then the problem might be approached using gain scheduling with $p^{*}$ playing the role of a scheduling variable but if the evolution of $p^{*}$ could not be determined, then the problem would typically call for an adaptive solution. This paper is concerned with this situation.

\section{Model-based control}

Assume that one has chosen a family of off-theshelf, candidate loop-controllers $\mathscr{C} \triangleq\left\{\mathbb{C}_{p}: p \in \mathscr{P}\right\}$, in such a way that for each $p \in \mathscr{P}, \mathbb{C}_{p}$ would "solve" the regulation problem, where $\mathbb{P}$ is any element of $\mathscr{F}_{p}$. 


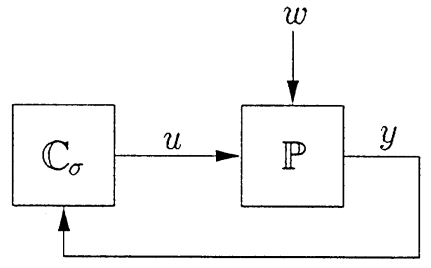

Fig. 1. Process and multi-controller feedback loop.

The idea then is to generate a switching signal $\sigma$ taking values in $\mathscr{P}$, which causes the output $y$ of the process model $\mathbb{P}$ in closed-loop with $\mathbb{C}_{\sigma}$, as shown in Fig. 1 , to be regulated about zero. We call $\mathbb{C}_{\sigma}$ a multi-controller and we require it to be a dynamical system with a real, finite-dimensional state space $\mathscr{X}_{\mathbb{C}}$ and defining equations of the form

$\dot{x}_{\mathbb{C}}=F_{\sigma}\left(x_{\mathbb{C}}, y\right), \quad u=G_{\sigma}\left(x_{\mathbb{C}}, y\right)$,

where for each fixed $p \in \mathscr{P}$, the equations

$\dot{\bar{x}}_{\mathbb{C}}=F_{p}\left(\bar{x}_{\mathbb{C}}, y\right), \quad u_{p}=G_{p}\left(\bar{x}_{\mathbb{C}}, y\right)$

model $\mathbb{C}_{p}$ and the $F_{p}$ and $G_{p}$ are locally Lipschitz continuous functions on $\mathscr{X}_{\mathbb{C}} \oplus \mathscr{Y}$. Thus we are implicitly assuming that all $\mathbb{C}_{p}$ have the same finite dimension. This is in fact a very mild assumption, since for $p \in \mathscr{P}, \mathbb{C}_{p}$ is not required to be "observable" through $u_{p}$. For example, if one initially had two controllers - say $\mathbb{C}_{p}$ and $\mathbb{C}_{q}$ - with the dimension of $\mathbb{C}_{p}$ less than that of $\mathbb{C}_{q}$, one could increase the dimension of $\mathbb{C}_{p}$ to match that of $\mathbb{C}_{q}$ by simply adjoining to $\mathbb{C}_{p}$ any stable subsystem $\Sigma$ of dimension $\operatorname{dim}\left(\mathbb{C}_{q}\right)-\operatorname{dim}\left(\mathbb{C}_{p}\right)$, in such a way that $\Sigma$ has no influence on $\mathbb{C}_{p}$ 's input-output behavior.

Depending on the way in which $\sigma$ is varied $\{$ which might be in either a piecewise-continuous or even a piecewise-constant manner $\}$, the algorithm which generates $\sigma$ might be called either a "tuner" or a "supervisor". In the sequel, we use the term supervisor and we restrict $\sigma$ to be piecewise-constant since this case is somewhat easier to understand. Much of which follows however, is also valid for piecewise-continuously tuned $\sigma$.

The types of supervisors which seem to be the most promising are those which utilize "estimators" and base controller selection on certainty equivalence. As mentioned before, certainty equivalence is a heuristic idea which advocates that in an adaptive context, the feedback control applied to an imprecisely modeled process should, at each instant of time, be designed on the basis of a current estimate of what the process is, with the understanding that each such estimate is to be viewed as correct even though it may not be. The heuristic is usually justified by reasoning that if process model estimates converge to the actual process model, then a certainty equivalence based controller ought to converge to the nonadaptive controller which would have been implemented had the correct process model been known in the first place. The problem with this justification is that process model estimates do not typically converge to the actual process model because of noise and unmodelled dynamics. So why then does certainty equivalence prove to be especially useful in an adaptive context? To answer this question we need the notion of "input-to-state stability" and an appropriate definition of detectability for nonlinear systems. These ideas are discussed next.

\section{Input-to-state stability and detectability}

Let

$\dot{x}=A(x, u), \quad y=C(x, u)$

be a finite-dimensional dynamical system whose state, input, and output take values in real, finite-dimensional spaces $\mathscr{X}, \mathscr{U}$, and $\mathscr{Y}$, respectively. Suppose that $A$ and $C$ are at least locally Lipschitz continuous on $\mathscr{X} \oplus \mathscr{U}$ and that $\tilde{u}$ is an equilibrium input of $A$; i.e., a fixed vector in $\mathscr{U}$ satisfying $A(\tilde{x}, \tilde{u})=0$ for some $\tilde{x} \in \mathscr{X}$. The following definition extends to nonzero equilibrium inputs, the concept of "input-to-state stability" given in [16].

Definition 1 (Input-to-state stability). The system defined by Eq. (2) \{or just the state dynamic $\dot{x}=A(x, u)$, or even just $A\}$ is said to be input-to-state stable about $\tilde{u}$, if $A(\tilde{x}, \tilde{u})=0$ for some state $\tilde{x} \in \mathscr{X}$, and there exist continuous, positive-definite, radially unbounded functions $V, X: \mathscr{X} \rightarrow[0, \infty), U: \mathscr{U} \rightarrow[0, \infty)$ such that $V$ is continuously differentiable, $V(0)=0, X(0)=0, U(0)=0$, and

$$
\begin{gathered}
\frac{\partial V(x-\tilde{x})}{\partial x} A(x, u) \leqslant-X(x-\tilde{x})+U(u-\tilde{u}), \\
\forall\{x, u\} \in \mathscr{X} \oplus \mathscr{U} .
\end{gathered}
$$

It is not difficult to prove that for each equilibrium input $\tilde{u} \in \mathscr{U}$ about which Eq. (2) is input-to-state stable, there can be only one state $\tilde{x} \in \mathscr{X}$ at which $A(\tilde{x}, \tilde{u})=0$. We call $\tilde{x}$ the equilibrium state induced by $\tilde{u}$. In the event that Eq. (2) is input-to-state stable about each of 
its equilibrium inputs, we say that Eq. (2) is input-tostate stable. In the sequel we call any list of functions $\{V, X, U\}$ with the aforementioned properties, a stability triple for Eq. (2) about $\tilde{u}$. Thus Eq. (2) is inputto-state stable about $\tilde{u}$ just in case $\tilde{u}$ is an equilibrium input of Eq. (2) and Eq. (2) possesses a stability triple about $\tilde{u}$. As defined, input-to-state stability implies that if $u$ is bounded then so is $x$ and if $u(t)=\tilde{u}, t \geqslant 0$, then $x \rightarrow \tilde{x}$ as $t \rightarrow \infty$. A more precise statement of these implications, for the case $\tilde{u}=0$, can be found in [15].

It is possible to define detectability in a number of different ways (see [17] and references therein). An especially useful characterization is in terms of an inequality much like Eq. (3). A definition along these lines, for systems without inputs, appears in [8]. A generalization of this definition which is particularly well-suited to our application, appears in [14] and is as follows.

Definition 2 (Detectability). The system defined by Eq. (2) $\{$ or just the pair $(C, A)\}$ is said to be detectable about an input-output pair $\{\tilde{u}, \tilde{y}\} \in \mathscr{U} \oplus \mathscr{Y}$ if $A(\tilde{x}, \tilde{u})=0$ and $\tilde{y}=C(\tilde{x}, \tilde{u})$ for some state $\tilde{x} \in \mathscr{X}$, and there exist continuous, positive-definite, radially unbounded functions $V, X: \mathscr{X} \rightarrow[0, \infty), U: \mathscr{U} \rightarrow[0, \infty)$, $Y: \mathscr{Y} \rightarrow[0, \infty)$ such that $V$ is continuously differentiable, $V(0)=0, X(0)=0, U(0)=0, Y(0)=0$ and

$$
\begin{aligned}
\frac{\partial V(x-\tilde{x})}{\partial x} A(x, u) \leqslant & -X(x-\tilde{x})+Y(C(x, u)-\tilde{y}) \\
& +U(u-\tilde{u}), \quad \forall\{x, u\} \in \mathscr{X} \oplus \mathscr{U} .
\end{aligned}
$$

Any pair $\{\tilde{u}, \tilde{y}\} \in \mathscr{U} \oplus \mathscr{Y}$ for which there exists a state $\tilde{x}$ such that $A(\tilde{x}, \tilde{u})=0$ and $\tilde{y}=C(\tilde{x}, \tilde{u})$, is said to be an equilibrium input-output pair of Eq. (2). It can be shown that for each equilibrium input-output pair $\{\tilde{u}, \tilde{y}\}$ about which Eq. (2) is detectable there is exactly one state $\tilde{x} \in \mathscr{X}$ at which $A(\tilde{x}, \tilde{u})=0$ and $\tilde{y}=C(\tilde{x}, \tilde{u})$. We call $\tilde{x}$ the equilibrium state induced by $\{\tilde{u}, \tilde{y}\}$. In addition, we call any list of functions $\{V, X, U, Y\}$ with the aforementioned properties, a detectability quadruple for Eq. (2) about $\{\tilde{u}, \tilde{y}\}$. Thus Eq. (2) is detectable about $\{\tilde{u}, \tilde{y}\}$ just in case $\{\tilde{u}, \tilde{y}\}$ is an equilibrium input-output pair and Eq. (2) possesses a detectability quadruple about $\{\tilde{u}, \tilde{y}\}$. In case Eq. (4) holds without the term $U(u-\tilde{u})$, Eq. (2) is said to be strongly detectable about $\tilde{y}$ and the list of functions $\{V, X, Y\}$ is called a strong detectability triple about $\tilde{y}$. We call any vector $\tilde{y} \in \mathscr{Y}$ an equilibrium output of Eq. (2) if for some $\tilde{u} \in \mathscr{U},\{\tilde{u}, \tilde{y}\}$ is an equilibrium input-output pair of Eq. (2). Clearly strong detectability implies detectability. It can be shown that for each equilibrium output $\tilde{y}$ about which Eq. (2) is strongly detectable there is exactly one state $\tilde{x} \in \mathscr{X}$ at which $A(\tilde{x}, \tilde{u})=0$ and $\tilde{y}=C(\tilde{x}, \tilde{u})$, for some $\tilde{u}$. We call $\tilde{x}$ the equilibrium state induced by the equilibrium output $\tilde{y}$.

The preceding definition of detectability reduces to the familiar one in the event that Eq. (2) is a linear system and $\tilde{u}$ and $\tilde{y}$ are both zero. In addition, the definition proves to capture the intuitive notion of detectability, namely that if $u$ and $y$ are bounded, then so is $x$ and if $u$ and $y$ converge to $\tilde{u}$ and $\tilde{y}$, respectively, then $x$ converges to $\tilde{x}$. More precisely, we can state the following lemma [17].

Lemma 1. Suppose Eq. (2) is detectable about an equilibrium input-output pair $\{\tilde{u}, \tilde{y}\}$ and that $\tilde{x}$ is the equilibrium state of Eq. (2) induced by this pair. There exist continuous, positive-definite, strictly monotone increasing functions $\gamma_{y}:[0, \infty)$ $\rightarrow[0, \infty), \quad \gamma_{u}:[0, \infty) \rightarrow[0, \infty)$, and a continuous, positive-definite function $\beta:[0, \infty) \times[0, \infty) \rightarrow[0, \infty)$ which is strictly monotone increasing on the second argument, and has $\lim _{\tau \rightarrow \infty} \beta(s, \tau)=0$ for each fixed $s \geqslant 0$, such that the following is true. For each initial state $x(0) \in \mathscr{X}$ and each piecewise-continuous signal $u$,

$$
\begin{aligned}
& \|x(t)-\tilde{x}\| \\
& \leqslant \beta\left(\left\|x\left(t_{0}\right)-\tilde{x}\right\|, t-t_{0}\right)+\gamma_{y}\left(\sup _{\tau \in\left[t_{0}, t\right]}\|y(\tau)-\tilde{y}\|\right) \\
& \quad+\gamma_{u}\left(\sup _{\tau \in\left[t_{0}, t\right]}\|u(\tau)-\tilde{u}\|\right), \quad t \geqslant t_{0} \geqslant 0
\end{aligned}
$$

along the corresponding solution to Eq. (2).

If $\{V, X, U\}$ is a stability triple for Eq. (2) about $\tilde{u}$, and $Y$ is any scalar-valued, locally Lipschitz continuous, positive-definite function, then $\{V, X, U, Y\}$ is a detectability quadruple for Eq. (2) about $\{\tilde{u}, C(\tilde{x}, \tilde{u})\}$ where $\tilde{x}$ is the equilibrium state of Eq. (2) induced by $\tilde{u}$; thus input-to-state stability about $\tilde{u}$ implies detectability about $\{\tilde{u}, C(\tilde{x}, \tilde{u})\}$. Even more is true if the point at which $u$ enters Eq. (2) is an injection channel; i.e., if $\mathscr{U}=\mathscr{Y}$ and there exists a locally 
Lipschitz continuous function $C^{-1}: \mathscr{X} \oplus \mathscr{Y} \rightarrow \mathscr{Y}$ such that $C\left(x, C^{-1}(x, u)\right)=u$ for all $u \in \mathscr{U}$ and all $x \in \mathscr{X}$.

Lemma 2. Suppose that the point at which u enters Eq. (2) is an injection channel and that Eq. (2) is input-to-state stable about an equilibrium input $\tilde{u}$. Then $\tilde{y} \triangleq \tilde{u}$ is an equilibrium output of the system

$\dot{x}=A(x, C(x, u)), \quad y=C(x, u)$

and Eq. (5) is strongly detectable about $\tilde{y}$. In addition, any stability triple for Eq. (2) about $\tilde{u}$ is a strong detectability triple for Eq. (5) about $\tilde{y}$. Moreover the equilibrium state of Eq. (5) induced by its equilibrium output $\tilde{y}$ is the same as the equilibrium state of Eq. (2) induced by its equilibrium input $\tilde{u}$.

Proof of Lemma 2. Set $\bar{u}=C^{-1}(\tilde{x}, \tilde{u})$ where $\tilde{x}$ is the equilibrium state of Eq. (2) induced by $\tilde{u}$. Since $\tilde{y}=\tilde{u}=C(\tilde{x}, \bar{u}) \quad$ and $\quad A(\tilde{x}, C(\tilde{x}, \tilde{u}))=A(\tilde{x}, \tilde{u})=0, \tilde{y}$ must be an equilibrium output of Eq. (5). From this and the fact that $A(x, C(x, u))=A(x, y)$, it follows that any stability triple for Eq. (2) about $\tilde{u}$ must be a strong detectability triple for Eq. (5) about $\tilde{y}$. Thus Eq. (5) must be strongly detectable about $\tilde{y}$ and $\tilde{x}$ must be the equilibrium state of Eq. (5) induced by $\tilde{y}$.

\section{Estimator-based supervisor}

An estimator-based supervisor consists of three subsystems, a "multi-estimator" $\mathbb{E}$, a performance signal generator $\mathbb{P} S$, and a switching logic $\mathbb{S}$ (see Fig. 2).

A multi-estimator is a parallel implementation of a family of "estimators", one for each $\mathbb{M}_{p} \in \mathscr{M}$. By an estimator $\mathbb{E}_{\mathbb{M}_{p}}$ for a given nominal process model $\mathbb{M}_{p}$, is meant any finite-dimensional, input-to-state stable dynamical system whose input is the pair $\{u, y\}$ and whose output is a signal $y_{p}$ which would be an asymptotically correct estimate of $y$, if $\mathbb{M}_{p}$ were the actual process model and there were no measurement noise or disturbances. For $\mathbb{E}_{\mathbb{M}_{p}}$ to have this property, it would have to exhibit under the feedback interconnection $y \triangleq y_{p}$ and an appropriate initialization $\}$ the same input-output behavior between $u$ and $y_{p}$ as $\mathbb{M}_{p}$ does between its input and output. ${ }^{3}$ For linear systems

\footnotetext{
${ }^{3}$ The main reason for not simply defining $\mathbb{E}_{\mathbb{M}_{p}} \triangleq \mathbb{M}_{p}$ is the requirement that $\mathbb{E}_{\mathbb{M}_{p}}$ be input-to-state stable. To require input-tostate stability of $\mathbb{M}_{p}$ would rule out open-loop unstable nominal process models which is too restrictive.
}

such estimators would typically be observers or identifiers [8]. Estimators can also be defined quite easily for certain types of nonlinear systems including those which are linearizable by output injection; in this category is any system whose state and measured output is one and the same.

By a multi-estimator $\mathbb{E}$ for a given family of nominal process models $\mathscr{M}=\left\{\mathbb{M}_{p}: p \in \mathscr{P}\right\}$ is meant a finite-dimensional, input-to-state stable system of the form

$\dot{x}_{\mathbb{E}}=A_{\mathbb{E}}\left(x_{\mathbb{E}}, u, y\right), \quad y_{p}=C_{p}\left(x_{\mathbb{E}}\right), \quad p \in \mathscr{P}$,

where, for each fixed $p \in \mathscr{P}$,

$\dot{x}_{\mathbb{E}}=A_{\mathbb{E}}\left(x_{\mathbb{E}}, u, y\right), \quad y_{p}=C_{p}\left(x_{\mathbb{E}}\right)$

is an estimator for $\mathbb{M}_{p}$. $\mathbb{E}$ is thus a parallel implementation of a family of estimators defined by Eq. (7) for each $p \in \mathscr{P}$. In the sequel, we write $\mathbb{E}_{p}$ for the $p$ th such estimator and $\mathscr{X}_{\mathbb{E}}$ for the state space of $\mathbb{E}$. We require $A_{\mathbb{E}}$ and the $C_{p}$ to be locally Lipschitz continuous on $\mathscr{X}_{\mathbb{E}} \oplus \mathscr{U} \oplus \mathscr{Y}$ and $\mathscr{X}_{\mathbb{E}}$, respectively. ${ }^{4}$

Note that all of the estimators implemented by $\mathbb{E}$, share the same state $x_{\mathbb{E}}$. The problem of constructing a family of state-sharing estimators $\left\{\mathbb{E}_{p}: p \in \mathscr{P}\right\}$ from a given family of estimators $\left\{\mathbb{E}_{\mathbb{M}_{p}}: p \in \mathscr{P}\right\}$ proves to be quite easy if $\mathscr{P}$ is a finite set. If $\mathscr{P}$ is not finite, such a construction can still be carried out under certain conditions. We will discuss this point further in Section 8.

A performance signal generator $\mathbb{P S}$ is a dynamical system whose inputs are output estimation errors $e_{p} \triangleq y_{p}-y, p \in \mathscr{P}$ and whose outputs are performance signals $\pi_{p}, p \in \mathscr{P}$. For each $p \in \mathscr{P}, \pi_{p}(t)$ is intended to be a suitably defined measure of the size of the $e_{p}$.

The third subsystem of an estimator-based supervisor is a switching logic $\mathbb{S}$. The role of $\mathbb{S}$ is to generate $\sigma$. Although there are many different ways to define $\mathbb{S}$, in each case the underlying strategy for generating $\sigma$ is more or less that same: From time to time set $\sigma$ equal to that value of $p \in \mathscr{P}$ for which $\pi_{p}$ is the smallest. ${ }^{5}$ The motivation for this idea is obvious: the

\footnotetext{
${ }^{4}$ The results which follow readily generalize to the case when the readout maps in Eq. (7) depend on $y$ \{i.e., when $y_{p}=$ $\left.C_{p}\left(x_{\mathbb{E}}, y\right)\right\}$ provided, for each such $C_{p}$, there is a map $\bar{C}_{p}: \mathscr{X}_{\mathbb{E}} \rightarrow \mathscr{Y}$ such that $\bar{C}_{p}\left(x_{\mathbb{E}}\right)=C\left(x_{\mathbb{E}}, \bar{C}_{p}\left(x_{\mathbb{E}}\right)\right)$ for all $x_{\mathbb{E}} \in \mathscr{X}_{\mathbb{E}}$. Readout maps depending on $y$ in this manner are sometimes useful in multioutput representations [11].

${ }^{5}$ Were we talking about a conventional adaptive control, $\mathscr{P}$ would typically be a continuum, there would be no performance signals, and in place of $\mathbb{S}$ there would be a tuning algorithm $\mathbb{T}$ driven by $e_{\sigma}$, which continuously tunes $\sigma$ according to some parameter adjustment rule.
} 


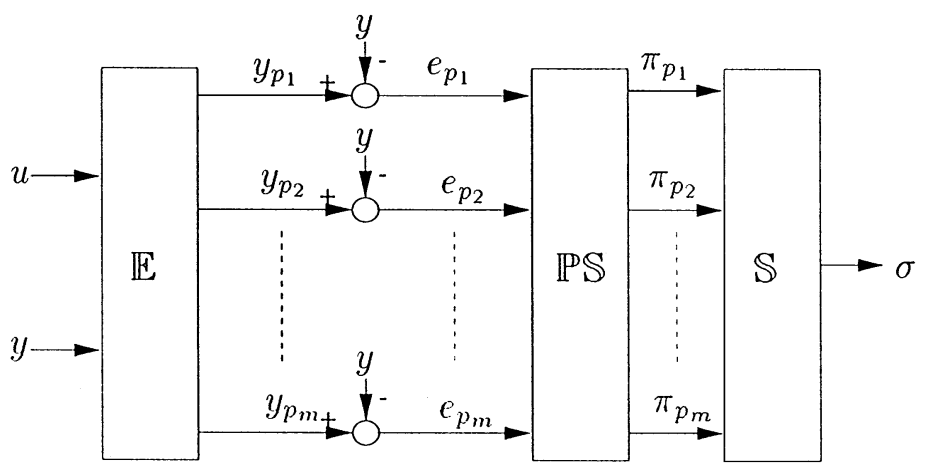

Fig. 2. Estimator-based supervisor.

nominal process model whose associated performance signal is the smallest, "best" approximates what the process is and thus the candidate controller designed on the basis of that model ought to be able to do the best job of controlling the process. The origin of this idea is the concept of certainty equivalence which we have discussed above.

\section{The implication of certainty equivalence}

To understand what certainty equivalence actually implies, let us assume that for each $p \in \mathscr{P}, \mathbb{C}_{p}$ has been chosen so that the system shown in Fig. 3 has $\tilde{v} \triangleq 0$ as an equilibrium input and that about this input the system is at least input-to-state stable. ${ }^{6}$ Suppose in addition, that $\bar{y}_{p}=0$ at the equilibrium state of this system induced by $\tilde{v}$.

By this we mean that for each $p \in \mathscr{P}$, the interconnected system

$$
\begin{aligned}
& \dot{\bar{x}}_{\mathbb{E}}=A_{\mathbb{E}}\left(\bar{x}_{\mathbb{E}}, \bar{u}_{p}, \bar{y}_{p}-v\right), \quad \bar{y}_{p}=C_{p}\left(\bar{x}_{\mathbb{E}}\right), \\
& \dot{\bar{x}}_{\mathbb{C}}=F_{p}\left(\bar{x}_{\mathbb{C}}, \bar{y}_{p}-v\right), \quad \bar{u}_{p}=G_{p}\left(\bar{x}_{\mathbb{C}}, \bar{y}_{p}-v\right)
\end{aligned}
$$

with input $v$, is input-to-state stable about an equilibrium input $\tilde{v}=0$, and moreover that at the equilibrium state induced by this input, $\bar{y}_{p}=0$. Justification for placing these requirements on $\mathbb{C}_{p}\{$ These requirements are implicit in most standard adaptive algorithms. \} stems first from the fact that the subsystem enclosed

\footnotetext{
${ }^{6}$ In practice, one would demand much more of the $\mathbb{C}_{p}$. For example, for each $p \in \mathscr{P}$ one would require the feedback connection of $\mathbb{C}_{p}$ with each model in $\mathscr{F}_{p}$, to be input-to-state stable with respect to $w$, about an equilibrium state at which the model's output is zero. The results which follows do not depend on this requirement.
}

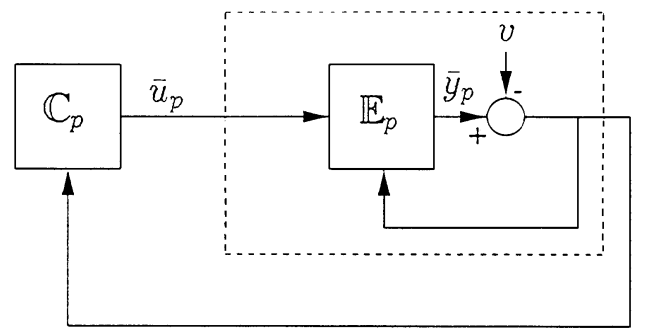

Fig. 3. Feedback interconnection.

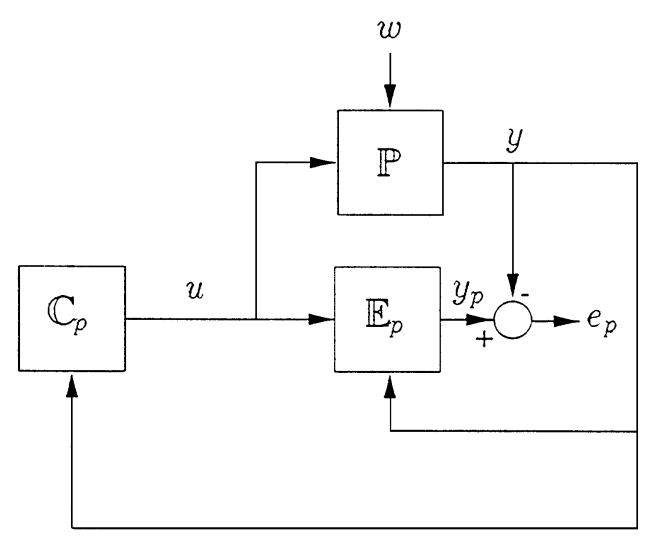

Fig. 4. $\Sigma_{p}$.

within the dashed box in Fig. 3 is input-output equivalent to $\mathbb{M}_{p}$ if $v \equiv 0$ and second from the control objective which is to regulate $y$ about zero. Here $v$ is to be regarded as a fictitious perturbing signal. In the sequel $v$ will be identified with $e_{p}$.

For each $p \in \mathscr{P}$, let $\Sigma_{p}$ then denote the system shown in Fig. 4 consisting of the interconnection of the process model $\mathbb{P}$, the estimator $\mathbb{E}_{p}$, and the controller $\mathbb{C}_{\sigma}$ with $\sigma$ held fixed at $p$. 
Thus $\Sigma_{p}$ is a dynamical system with input $w$ and output

$e_{p}=y_{p}-y$

defined by the process model

$\dot{x}_{\mathbb{P}}=A_{\mathbb{P}}\left(x_{\mathbb{P}}, w, u\right) \quad y=C_{\mathbb{P}}\left(x_{\mathbb{P}}, w\right)$

together with the $p$ th estimator-controller equations

$\dot{x}_{\mathbb{E}}=A_{\mathbb{E}}\left(x_{\mathbb{E}}, u, y\right), \quad y_{p}=C_{p}\left(x_{\mathbb{E}}\right)$,

$\dot{x}_{\mathbb{C}}=F_{p}\left(x_{\mathbb{C}}, y\right), \quad u=G_{p}\left(x_{\mathbb{C}}, y\right)$.

The main result of this paper is as follows.

Theorem 1 (Certainty equivalence stabilization). Let $\mathbb{P}$ be a process model in $\mathscr{F}$ which is detectable about each of its equilibrium input-output pairs. Let $p \in \mathscr{P}$ be fixed. The system $\Sigma_{p}$ shown in Fig. 4 and defined by Eqs. (9)-(11) is detectable about any equilibrium input-output pair $\left\{\tilde{w}, \tilde{e}_{p}\right\}$ it has, for which $\tilde{e}_{p}=0$.

A proof of this theorem is given in the appendix.

The implication of Theorem 1 is clear. For each process model $\mathbb{P} \in \mathscr{F}$ which is detectable about all of its equilibrium inputs, and for each $p \in \mathscr{P}$, the inputto-state stabilization of the system in Fig. 3 by $\mathbb{C}_{p}$ causes the system $\Sigma_{p}$ shown in Fig. 4 to be detectable about each equilibrium input-output pair $\left\{\tilde{w}, \tilde{e}_{p}\right\}$ it has $\{$ if any $\}$, for which $\tilde{e}_{p}=0$. This is what is meant by the phrase certainty equivalence implies detectability.

With the preceding in mind, recall the underlying decision-making strategy of an estimator-based supervisor: From time to time select for $\sigma$, that value $q \in \mathscr{P}$ such that the performance signal $\pi_{q}$ is the smallest among the $\pi_{p}, p \in \mathscr{P}$. Justification for this strategy is now clear: By choosing $\sigma$ to maintain smallness of $\pi_{\sigma}$ and consequently $e_{\sigma}$, the supervisor is also maintaining smallness of the composite state of the interconnection of $\mathbb{P}, \mathbb{C}_{\sigma}$, and $\mathbb{E}$ because of detectability through $e_{\sigma}$ for each fixed value of $\sigma$.

\section{Injected systems}

In this section, we digress to define and briefly discuss a useful family of systems which do not depend on $\mathbb{P}$ and which turn out to be detectable because of certainty equivalence. In particular, for each pair $p, q \in \mathscr{P}$, let

$\dot{\bar{x}}=A_{p q}(\bar{x}, v), \quad e_{p q}=C_{p q}(\bar{x})+v$ abbreviate the system with input $v$, state $\bar{x} \triangleq\left[\begin{array}{ll}\bar{x}_{\mathbb{E}}^{\prime} & \bar{x}_{\mathbb{C}}^{\prime}\end{array}\right]^{\prime}$ and output $e_{p q}=\bar{y}_{p}-\bar{y}_{q}+v$ defined by the equations

$\dot{\bar{x}}_{\mathbb{E}}=A_{\mathbb{E}}\left(\bar{x}_{\mathbb{E}}, \bar{u}_{p}, \bar{y}_{q}-v\right)$,

$\bar{y}_{l}=C_{l}\left(\bar{x}_{\mathbb{E}}\right), \quad l \in\{p, q\}$,

$\dot{\bar{x}}_{\mathbb{C}}=F_{p}\left(\bar{x}_{\mathbb{C}}, \bar{y}_{q}-v\right), \quad \bar{u}_{p}=G_{p}\left(\bar{x}_{\mathbb{C}}, \bar{y}_{q}-v\right)$.

Note that if $p=q$, the system defined by the differential equation (13), namely $\dot{x}=A_{p p}(\bar{x}, v)$, is the same as the system defined by Eq. (8). By assumption, the latter is input-to-state stable about $\tilde{v}=0$ and, in addition, $\bar{y}_{p}=0$ at the equilibrium state induced by this equilibrium input. Moreover, if $C(x, v) \triangleq C_{p q}(x)+v$, then $C^{-1}(x, v)=v-C_{p q}(x)$, so the point at which $v$ enters Eq. (8) is an injection channel. It follows from Lemma 2 that the system

$\dot{\bar{x}}=A_{p p}\left(\bar{x}, C_{p q}(\bar{x})+v\right), \quad e_{p q}=C_{p q}(\bar{x})+v$

is strongly detectable about the equilibrium output $\tilde{e}_{p q} \triangleq 0$, that the equilibrium state of Eq. (14) induced by this equilibrium output is the same as the equilibrium state of Eq. (13) induced by $\tilde{v}$, and that any stability triple for Eq. (13) about $\tilde{v}$ is a strong detectability triple for Eq. (14) about $\tilde{e}_{p q}$. Thus for any $p$ and $q$ in $\mathscr{P}$, Eq. (14) is strongly detectable about $\tilde{e}_{p q}=0$, and $\bar{y}_{p}=0$ at the equilibrium state of Eq. (14) induced by this output. Since for any fixed $p, q \in \mathscr{P}$, the definition of $A_{p q}$ implies that $A_{p q}(\bar{x}, v)=A_{p p}\left(\bar{x}, C_{p q}(\bar{x})+v\right)$, we can therefore conclude the following:

Theorem 2. For each $p, q \in \mathscr{P}$, the system described by Eq. (12), with input $v$ and output $e_{p q}$, is strongly detectable about the equilibrium output $\tilde{e}_{p q} \triangleq 0$. In addition, any stability triple about $\tilde{v}=0$ for the inputto-state stable system defined by Eq. (13) with $q \triangleq p$, is a strong detectability triple about $\tilde{e}_{p q}=0$ for Eq. (12) for all $q \in \mathscr{P}$. Moreover $\bar{y}_{p}=0$ at the equilibrium state induced by $\tilde{e}_{p q}$.

In analyzing adaptive and supervisory control systems, it is often convenient to focus attention on subsystems of the form

$$
\begin{aligned}
& \dot{x}_{\mathbb{E}}=A_{\mathbb{E}}\left(x_{\mathbb{E}}, u, y\right), \\
& e_{l}=C_{l}\left(x_{\mathbb{E}}\right)-y, \quad l \in\{p, q\}, \\
& \dot{x}_{\mathbb{C}}=F_{p}\left(x_{\mathbb{C}}, y\right), \quad u=G_{p}\left(x_{\mathbb{C}}, y\right),
\end{aligned}
$$

where $q$ is typically the index $p^{*}$ of the subfamily $\mathscr{F}_{p^{*}}$ within which $\mathbb{P}$ resides [9]. By the pqth injected system is meant the system which results when the equation $y=C_{q}\left(x_{\mathbb{E}}\right)-e_{q}$ from Eq. (15) is used to 
eliminate $y$ from $A_{\mathbb{E}}(\cdot), F_{p}(\cdot)$ and $G_{p}(\cdot)$ in Eq. (15). It is easy to see that once this is done, the $p q$ th injected system can be written as

$\dot{x}=A_{p q}\left(x, e_{q}\right), \quad e_{p}=C_{p q}(x)+e_{q}$,

where $x=\left[\begin{array}{ll}x_{\mathbb{E}}^{\prime} & x_{\mathbb{C}}^{\prime}\end{array}\right]^{\prime}$. We have at once the following.

Corollary 1. For each $p, q \in \mathscr{P}$, the pqth injected subsystem, namely Eq. (16), with input $e_{q}$ and output $e_{p}$, is strongly detectable about the equilibrium output $\tilde{e}_{p} \triangleq 0$. In addition, any stability triple about the equilibrium input $\tilde{v}=0$ of the input-to-state stable system $\dot{x}=A_{p p}(x, v)$, is a strong detectability triple about $\tilde{e}_{p}$ for Eq. (16). Moreover $y_{p}=0$ at the equilibrium state induced by $\tilde{e}_{p}$.

The advantage of working with the injected systems $\dot{x}=A_{p p^{*}}\left(x, e_{p^{*}}\right), e_{p}=C_{p p^{*}}(x)+e_{p^{*}}, p \in \mathscr{P}$, rather than the overall system comprised of $\mathbb{P}, \mathbb{C}_{\sigma}$ and $\mathbb{E}$, is that the maps defining the former, namely the $A_{p p^{*}}$ and $C_{p p^{*}}$, depend only on the subfamily $\mathscr{F}_{p^{*}}$ within which $\mathbb{P}$ resides and not on $\mathbb{P}$ itself. This is especially useful when one tries to take unmodeled dynamics into account.

\section{State sharing}

Let $\left\{\mathbb{E}_{\mathbb{M}_{p}}: p \in \mathscr{P}\right\}$ be a given family of estimators, each of the form

$\dot{z}_{p}=D_{p}\left(z_{p}, u, y\right), \quad \bar{y}_{p}=B_{p}\left(z_{p}\right)$,

where $z_{p}$ takes values in a real finite-dimensional space $\mathscr{Z}_{p}$ and $D_{p}$ and $B_{p}$ are locally Lipschitz continuous on $\mathscr{Z}_{p} \oplus \mathscr{U} \oplus \mathscr{Y}$ and $\mathscr{Z}_{p}$, respectively. Our aim is to explain how to construct a family of input-to-state stable, state-sharing estimators $\left\{\mathbb{E}_{p}: p \in \mathscr{P}\right\}$ in such a way that for each $p \in \mathscr{P}, \mathbb{E}_{p}$ and $\mathbb{E}_{\mathbb{M}_{p}}$ have the same input-output behavior. Each $\mathbb{E}_{p}$ is thus to be of the form

$\dot{x}_{\mathbb{E}}=A_{\mathbb{E}}\left(x_{\mathbb{E}}, u, y\right), \quad y_{p}=C_{p}\left(x_{\mathbb{E}}\right)$

with shared state space $\mathscr{X}_{\mathbb{E}}$. We require $A_{\mathbb{E}}$ and the $C_{p}$ to be locally Lipschitz continuous on $\mathscr{X}_{\mathbb{E}} \oplus \mathscr{U} \oplus \mathscr{Y}$ and $\mathscr{X}_{\mathbb{E}}$, respectively.

Suppose first that $\mathscr{P}$ is a finite set. It is then clearly possible to represent all of the estimators given by Eq. (17) together as a single dynamical system of the form (18) where $x_{\mathbb{E}} \triangleq \operatorname{column}\left\{z_{p}: p \in \mathscr{P}\right\}$ and $A_{\mathbb{E}}$ and the $C_{p}$ are locally Lipschitz continuous functions defined by

$$
\begin{aligned}
& A_{\mathbb{E}}\left(x_{\mathbb{E}}, u, y\right) \triangleq \operatorname{column}\left\{D_{p}\left(z_{p}, y, u\right): p \in \mathscr{P}\right\}, \\
& C_{p}\left(x_{\mathbb{E}}\right) \triangleq B_{p}\left(z_{p}\right), \quad p \in \mathscr{P} .
\end{aligned}
$$

In fact, for each fixed $p \in \mathscr{P}$, Eq. (18) has the same input-output behavior between $\{u, y\}$ and $y_{p}$ as $\mathbb{E}_{\mathbb{M}_{p}}$ does and is input-to-state stable. Indeed, as a stability triple for Eq. (18) about any given equilibrium input $\{\tilde{u}, \tilde{y}\}$, one could choose

$$
\left\{\sum_{p \in \mathscr{P}} V_{p}\left(z_{p}\right), \sum_{p \in \mathscr{P}} X_{p}\left(z_{p}\right), \sum_{p \in \mathscr{P}} U_{p}(y, u)\right\},
$$

where for each $p \in \mathscr{P},\left\{V_{p}, X_{p}, U_{p}\right\}$ is a stability triple for $\mathbb{E}_{\mathbb{M}_{p}}$ about $\{\tilde{u}, \tilde{y}\}$. In summary, any finite family of $\{$ parallel $\}$ estimators sometimes called "multiple models" $\}$ can be viewed as a single multi-estimator whose state is the composite of the states of the constitute estimators in the family.

If $\mathscr{P}$ is not a finite set and we were to define the $\mathbb{E}_{p}$ as above, then the $\mathbb{E}_{p}$ 's shared state space would clearly not be finite-dimensional. It turns out that it is possible to construct finite-dimensional, state-sharing estimators, for a given family $\left\{\mathbb{E}_{\mathbb{M}_{p}}: p \in \mathscr{P}\right\}$, even when $\mathscr{P}$ is not finite, provided all the $\mathbb{E}_{\mathbb{M}_{p}}$ are of the same dimension $n$ and the functions $D_{p}$ appearing in Eq. (17) are "affinely separable". The $D_{p}$ are affinely separable if there is a constant $n \times n$ stability matrix $D$, a positive integer $m$, a locally Lipschitz continuous function $R: \mathscr{U} \oplus \mathscr{Y} \rightarrow \mathbb{R}^{n \times m}$, and a vector-valued function $p \mapsto k_{p}$ from $\mathscr{P}$ to $\mathbb{R}^{m}$ such that

$D_{p}\left(z_{p}, u, y\right)=D z_{p}+R(u, y) k_{p}$.

Under these conditions the multi-estimator maps appearing in Eq. (18) might take the form

$A_{\mathbb{E}}\left(x_{\mathbb{E}}, u, y\right) \triangleq \bar{D} x_{\mathbb{E}}+\operatorname{stack}\{R(x, y)\}$,

$C_{p}\left(x_{\mathbb{E}}\right) \triangleq B_{p}\left(K_{p} x_{\mathbb{E}}\right), \quad p \in \mathscr{P}$,

where $x_{\mathbb{E}} \in \mathbb{R}^{n m}, \quad \bar{D} \triangleq$ block diagonal $\{\overbrace{D, \ldots, D}^{m \text { times }}\}$, $K_{p} \triangleq\left[\begin{array}{llll}k_{p 1} I_{n \times n} & k_{p 2} I_{n \times n} & \cdots & k_{p m} I_{n \times n}\end{array}\right], k_{p i}$ is the $i$ th element of $k_{p}$, and $\operatorname{stack}\{R(u, y)\}$ is the $n m$-vector which results when the $m$ columns of $R(u, y)$ are stack one atop the next. It is not difficult to show with $A_{\mathbb{E}}$ so defined, that for each input $\{u, y\}$, the state error $z_{p}(t)-K_{p} x_{\mathbb{E}}(t)=\mathrm{e}^{D t}\left(z_{p}(0)-K_{p} x_{\mathbb{E}}(0)\right), p \in \mathscr{P}$. This, the definitions of the $C_{p}$, and the Lipschitz 
continuity of the $B_{p}$ can then be used to verify that $\bar{y}_{p}(t)-y_{p}(t) \rightarrow 0, p \in \mathscr{P}$, as $t \rightarrow \infty$. In this sense, Eq. (18) has the same input-output behavior between $\{u, y\}$ and $y_{p}$ as $\mathbb{E}_{\mathbb{M}_{p}}$ does. Moreover, Eq. (18) is input-to-state stable because $\bar{D}$ is a stability matrix.

The preceding constructions are given for illustrative purposes only. In many cases one can exploit the detailed structure of the $\mathbb{E}_{\mathbb{M}_{p}}\{$ e.g., linearity $\}$ to obtain more appealing $\{$ e.g., lower dimensional $\} \mathbb{E}_{p}$.

\section{Exact matching}

The aim of this section is to briefly illustrate how the preceding results might be used to deduce global boundedness and asymptotic convergence in a supervisory control system when $\mathscr{P}$ is a finite, ordered set - say $\mathscr{P} \triangleq\{1,2, \ldots, m\}$. We shall do this only for the special case when $w=0$ and one of the $y_{p}$ is an asymptotically correct estimate of $y$. The equations for $\mathbb{P}, \mathbb{E}$, and $\mathbb{C}_{\sigma}$ are

$\dot{x}_{\mathbb{P}}=A_{\mathbb{P}}\left(x_{\mathbb{P}}, 0, u\right), \quad y=C_{\mathbb{P}}\left(x_{\mathbb{P}}\right)$,

$\dot{x}_{\mathbb{E}}=A_{\mathbb{E}}\left(x_{\mathbb{E}}, u, y\right), \quad e_{p}=C_{p}\left(x_{\mathbb{E}}\right)-y, p \in \mathscr{P}$

and

$\dot{x}_{\mathbb{C}}=F_{\sigma}\left(x_{\mathbb{C}}, y\right), \quad u=G_{\sigma}\left(x_{\mathbb{C}}, y\right)$,

respectively where $A_{\mathbb{P}}, A_{\mathbb{E}}$, and $C_{\mathbb{P}}$, as well as the $C_{p}, F_{p}$, and $G_{p}, p \in \mathscr{P}$, are at least locally Lipschitz continuous functions.

Corollary 1 guarantees that for any $p, q \in \mathscr{P}$, the $p q$ th injected system

$\dot{x}=A_{p q}\left(x, e_{q}\right), \quad e_{p}=C_{p q}(x)+e_{q}$

determined by Eqs. (21) $-(23)$ with $\sigma$ frozen at $p$ and

$x \triangleq\left[\begin{array}{l}x_{\mathbb{E}} \\ x_{\mathbb{C}}\end{array}\right]$,

is strongly detectable about the equilibrium output $\tilde{e}_{p} \triangleq 0$ and, in addition, $e_{q}=0$ at the equilibrium state induced by this output. For each such $p$, let $\left\{V_{p}, X_{p}, Y_{p}\right\}$ be a stability triple for $\dot{x}=A_{p p}(x, v)$ about the equilibrium input $\tilde{v}=0$. Corollary 1 also guarantees that $\left\{V_{p}, X_{p}, Y_{p}\right\}$ is a strong detectability triple for Eq. (24) about its equilibrium output 0 .
In the sequel we will consider exponentially weighted performance signals of the form

$\dot{\pi}_{p}=-\lambda \pi_{p}+Y_{p}\left(e_{p}\right), \quad \pi_{p}(0)>0, \quad p \in \mathscr{P}$,

where $\lambda$ is a prespecified positive number.

We shall make three assumptions.

Assumption 1. Each process model in $\mathscr{F}$ is detectable about at least one equilibrium input-output pair.

With Assumption 1, Lemma 1 allows one to conclude that if $u$ and $y$ are bounded then so is the state $x_{\mathbb{P}}$ of the process.

Assumption 2. Each $Y_{p}$ is continuously differentiable.

Assumption 3. There exists an index $p^{*} \in \mathscr{P}$ such that, for each initial state $\left\{x_{\mathbb{P}}(0), x_{\mathbb{E}}(0)\right\} \in \mathscr{X}_{\mathbb{P}} \oplus \mathscr{X}_{\mathbb{E}}$ and each piecewise-continuous, open-loop control signal $u,\left\|e_{p^{*}}\right\|$ and

$\int_{0}^{t} \mathrm{e}^{\lambda \tau}\left\|e_{p^{*}}(\tau)\right\| \mathrm{d} \tau$

are bounded on the interval of maximal length on which a solution to Eqs. (21) and (22) exists.

$\mathbb{E}$ can typically be constructed so that this assumption is satisfied in the noise/disturbance free case, provided $\mathbb{P}$ is input-output equivalent to a nominal model \{say $\mathbb{M}_{p^{*}}$ \} which is linearizable by output injection.

The implication of Assumptions 2 and 3 is that for any initialization of Eqs. (21)-(23) and (26), and any piecewise-constant signal $s:[0, \infty) \rightarrow \mathscr{P}$,

$\int_{0}^{T_{s}} \mathrm{e}^{\lambda t} Y\left(e_{p^{*}}\right) \mathrm{d} t<\infty$,

where $\left[0, T_{S}\right)$ is the interval of maximal length on which there exists a solution to Eqs. (21)-(23) and (26) when $\sigma \triangleq s$. Use will be made of this property in the sequel.

For $\mathbb{S}$ we consider what we shall call "scaleindependent hysteresis switching". By a scaleindependent hysteresis switching logic is meant a hybrid dynamical sytem $\mathbb{S} \triangleq \mathbb{S}_{\mathbb{\sharp}}$ whose inputs are the $\pi_{p}$ and whose state and output are both $\sigma$. To specify $\mathbb{S}_{\mathbb{H}}$ it is necessary to first pick a positive number $h>0$ called a hysteresis constant. $\mathbb{S}_{\sharp}$ 's internal logic is then defined by the computer diagram shown in Fig. 5, where at each time $t, q$ is the smallest element in $\mathscr{P}$ which minimizes $\pi_{p}$. 


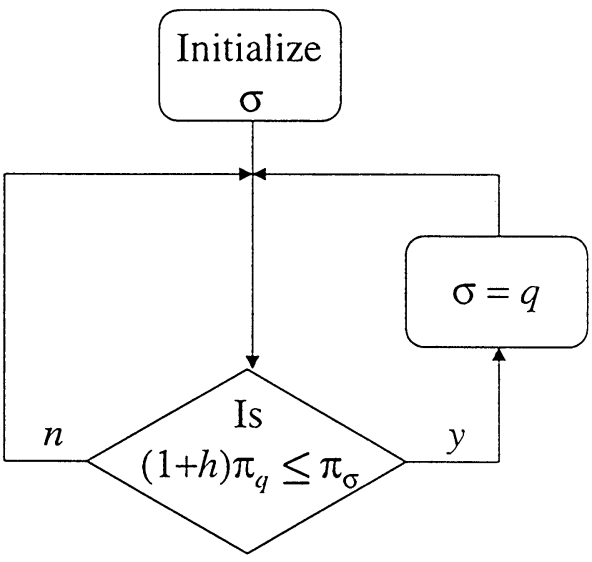

Fig. 5. Computer diagram of $\mathbb{S}_{\mathbb{H}}$.

In interpreting this diagram it is to be understood that $\sigma$ 's value at each of its switching times $\bar{t}$ is its limit from above as $t \downarrow \bar{t}$. Thus if $\bar{t}_{i}$ and $\bar{t}_{i+1}$ are any two successive switching times, then $\sigma$ is constant on $\left[\bar{t}_{i}, \bar{t}_{1+i}\right)$. The functioning of $\mathbb{S}_{\mathbb{W}}$ is roughly as follows. Suppose that at some time $t_{0}, \mathbb{S}_{\mathbb{M}}$ has just changed the value of $\sigma$ to $q$. $\sigma$ is then held fixed at this value unless and until there is a time $t_{1}>t_{0}$ at which $(1+h) \pi_{p} \leqslant \pi_{p}$ for some $p \in \mathscr{P}$. If this occurs, $\sigma$ is set equal to $p$ and so on.

The term "scale independence" is prompted by the fact that if $\theta$ is any continuous signal which is positive on $[0, \infty)$, then the response $\sigma$ of $\mathbb{S}_{\mathbb{\sharp}}$ will remain unchanged if its inputs, namely the $\pi_{p}, p \in \mathscr{P}$, are replaced with the "scaled performance signals" $\theta \pi_{p}, p \in \mathscr{P}$. This is because for such $\theta,(1+h) \pi_{p} \leqslant \pi_{q}$ and $\theta(1+h) \pi_{p} \leqslant \theta \pi_{q}$ are equivalent inequalities for all $p, q \in \mathscr{P}$ and all $t \in[0, \infty)$.

With the preceding in mind, let us define the scaled performance signals $\bar{\pi}_{p} \triangleq \mathrm{e}^{\lambda t} \pi_{p}, p \in \mathscr{P}$. In view of the definitions of the $\pi_{p}$ in Eq. (26), the $\bar{\pi}_{p}$ satisfy

$\bar{\pi}_{p}=\pi_{p}(0)+\int_{0}^{t} \mathrm{e}^{\lambda \tau} Y\left(e_{p}\right) \mathrm{d} \tau, \quad p \in \mathscr{P}$.

From this and Eq. (27) it follows that for any initialization of Eqs. (21) $-(23)$ and (26), and any piecewiseconstant signal $s:[0, \infty) \rightarrow \mathscr{P}$, (i) $\bar{\pi}_{p^{*}}$ will be bounded on $\left[0, T_{s}\right)$; moreover because they are monotone signals on any such interval, (ii) each $\bar{\pi}_{p}$ will have a limit as $t \rightarrow T_{s}$. The fact that the $\bar{\pi}_{p}$ possess these two properties will be made use of in the sequel.

Let us note that the interconnected system defined by Eqs. (21)-(23) and (26) is a dynamical system of the form

$\dot{z}=f_{\sigma}(z, t) \quad \pi_{p}=g_{p}(z), \quad p \in \mathscr{P}$,

where $z \triangleq\left\{x_{\mathbb{P}}, x_{\mathbb{E}}, x_{\mathbb{C}}, \pi_{1}, \pi_{2}, \ldots, \pi_{m}\right\}$ and for $p \in \mathscr{P}$, $f_{p}$ and $g_{p}$ are locally Lipschitz. Because of the hysteresis constant $h$, for each initial state $\{z(0) ; \sigma(0)\}$ there must be an interval $[0, T)$ of maximal length on which there is a unique pair $\{z ; \sigma\}$ with $z$ continuous and $\sigma$ piecewise constant, which satisfies Eq. (29) assuming $\sigma$ is generated by $\mathbb{S}_{\mathbb{W}}$ [5]. Moreover, on each proper subinterval $[0, \tau) \subset[0, T), \sigma$ can switch at most a finite number of times.

The fact that the $\bar{\pi}_{p}$ possess properties (i) and (ii) noted above, enable us to exploit the ScaleIndependent Hysteresis Switching Lemma [5] and consequently to draw the following conclusion.

Lemma 3. For fixed initial states $x_{\mathbb{P}}(0) \in \mathscr{X}_{\mathbb{P}}$, $x_{\mathbb{E}}(0) \in \mathscr{X}_{\mathbb{E}}, x_{\mathbb{C}}(0) \in \mathscr{X}_{\mathbb{C}}, \pi_{p}(0)>0 . p \in \mathscr{P}, \sigma(0) \in \mathscr{P}$, let $\left\{x_{\mathbb{P}}, x_{\mathbb{E}}, x_{\mathbb{C}}, \pi_{1}, \pi_{2}, \ldots, \pi_{m}, \sigma\right\}$ denote the unique solution to Eqs. (21)-(23) and (26) with $\sigma$ the output of $\mathbb{S}_{\mathbb{⿴}}$ - and suppose $[0, T)$ is the largest interval on which this solution is defined. There is a time $T^{*}<T$ beyond which $\sigma$ is constant and no more switching occurs. In addition, the scaled performance signal $\mathrm{e}^{\lambda t} \pi_{\sigma\left(T^{*}\right)}$ is bounded on $[0, T)$.

Let $x_{\mathbb{P}}, x_{\mathbb{E}}, x_{\mathbb{C}}, \pi_{1}, \pi_{2}, \ldots, \pi_{m}, \sigma, T$ and $T^{*}$ be as in Lemma 3 and set $q^{*} \triangleq \sigma\left(T^{*}\right)$. In view of Eqs. (27), (28) and the observation that $\mathrm{e}^{\lambda t} \pi_{q^{*}}$ must be bounded on $[0, T)$,

$\int_{0}^{T} Y_{q^{*}}\left(e_{q^{*}}(\tau)\right) \mathrm{d} \tau<\infty$.

Since $\sigma$ is frozen at $q^{*}$ for $t \in\left[T^{*}, T\right)$ and $\left\{V_{q^{*}}, X_{q^{*}}\right.$, $\left.Y_{q^{*}}\right\}$ is a strong detectability triple for Eq. (24) \{with $\left.p \triangleq q^{*}\right\}$ about the equilibrium output $\tilde{e}_{q^{*}} \triangleq 0$, we can write

$\dot{V}_{q^{*}}(x-\tilde{x}) \leqslant-X_{q^{*}}(x-\tilde{x})+Y_{q^{*}}\left(e_{q^{*}}\right), \quad t \in\left[T^{*}, T\right)$,

where $\tilde{x}$ is the equilibrium state of Eq. (24) \{with $\left.p \triangleq q^{*}\right\}$ induced by $\tilde{e}_{q^{*}}$. Therefore, by integrating the preceding we obtain

$$
\begin{aligned}
& V_{q^{*}}(x(t)-\tilde{x}) \leqslant V_{q^{*}}\left(x\left(t_{0}\right)-\tilde{x}\right)-\int_{t_{0}}^{t} X_{q^{*}}(x(\tau)-\tilde{x}) \mathrm{d} \tau \\
& \quad+\int_{t_{0}}^{t} Y_{q^{*}}\left(e_{q^{*}}(\tau)\right) \mathrm{d} \tau, \quad T^{*} \leqslant t_{0} \leqslant t \leqslant T
\end{aligned}
$$


From this and Eq. (30) it follows that $V_{q^{*}}(x(t)-\tilde{x})<\infty$ for $T^{*} \leqslant t_{0} \leqslant t<T$. Thus $V_{q^{*}}(x(t)-\tilde{x})$ is bounded on $\left[T^{*}, T\right)$ and consequently on $[0, T)$. But $V_{q^{*}}(\cdot)$ is radially unbounded, so $x$ and consequently $x_{\mathbb{E}}$ and $x_{\mathbb{C}}$ \{cf., Eq. (25) $\}$ must be bounded on $[0, T)$ as well.

In view of Eq. (22), $y=e_{p^{*}}+C_{p^{*}}\left(x_{\mathbb{E}}\right)$. By Assumption $3, e_{p^{*}}$ is bounded on $[0, T)$, so $y$ must also be. Boundedness of $u$ on $[0, T)$ then follows from the formula $u=G_{\sigma}\left(x_{\mathbb{C}}, y\right)$. Therefore $x_{\mathbb{P}}$ is bounded on $[0, T)$ because of Assumption 1 and Lemma 1. So is each $e_{p}, p \in \mathscr{P}$ because of the defining formula $e_{p}=C_{p}\left(x_{\mathbb{E}}\right)-y$. Therefore, each $\pi_{p}$ will be bounded on $[0, T)$ because the differential equations (26) defining the $\pi_{p}$ are input-to-state stable systems and the $Y_{p}\left(e_{p}\right)$ are bounded signals on $[0, T)$. In other words $x_{\mathbb{P}}, x_{\mathbb{E}}, x_{\mathbb{C}}$, and the $\pi_{p}$ are all bounded on $[0, T)$.

Now if $T$ were finite, the solution to Eqs. (21)(23) and (26) could be continued onto at least an open half interval of the form $\left[T, T_{1}\right)$ thereby contradicting the hypothesis that $[0, T)$ is the system's interval of maximal existence. By contradiction one can therefore conclude that $T=\infty$ and that $x_{\mathbb{P}}, x_{\mathbb{E}}, x_{\mathbb{C}}$ and the $\pi_{p}$ are bounded on $[0, \infty)$.

It will now be shown that $x$ converges to $\tilde{x}$ as $t \rightarrow \infty$. Define

$\bar{V}(t) \triangleq V_{q^{*}}(x(t)-\tilde{x})+\int_{t}^{\infty} Y_{q^{*}}\left(e_{q^{*}}\right) \mathrm{d} \tau, \quad t \geqslant T^{*}$.

Then $\bar{V}$ is a nonnegative bounded function. Moreover, from Eq. (31), $\dot{V} \leqslant-X_{q^{*}}(x-\tilde{x}), t \geqslant T^{*}$ so $\bar{V}$ is nonincreasing on $\left[T^{*}, \infty\right)$. We claim that $\bar{V} \rightarrow 0$ as $t \rightarrow \infty$. Suppose this were not so. Then there would be a positive number $\mu$ such that $\bar{V}(t) \geqslant 2 \mu$ for $t \geqslant T^{*}$. Pick $t_{1} \geqslant T^{*}$ so large that

$\int_{t}^{\infty} Y_{q^{*}}\left(e_{q^{*}}\right) \mathrm{d} \tau \leqslant \mu$.

Then $V_{q^{*}}(x(t)-\tilde{x}) \geqslant \mu$ for $t \geqslant t_{1}$. But $V_{q^{*}}(\cdot)$ is positivedefinite so there would have to be a positive number $\bar{\mu}$ such that $\|x(t)-\tilde{x}\| \geqslant \bar{\mu}, t \geqslant t_{1}$. As $X_{q^{*}}(\cdot)$ is also positive-definite, there would have to be another positive number $\delta$ such that $X_{q^{*}}(x(t)-\tilde{x}) \geqslant \delta, t \geqslant t_{1}$. But then one would have that $\dot{\bar{V}} \leqslant-\delta, t \geqslant t_{1}$ and thus that $\bar{V}(t)-\bar{V}\left(t_{1}\right) \leqslant-\delta\left(t-t_{1}\right) t \geqslant t_{1}$. Hence for $t$ sufficiently large $\bar{V}$ would become negative which is impossible. Therefore $\bar{V} \rightarrow 0$ as $t \rightarrow \infty$. From this it follows that $\lim _{t \rightarrow \infty} V_{q^{*}}(x(t)-\tilde{x})=0$. This proves that $x \rightarrow \tilde{x}$ as $t \rightarrow \infty$ since $V_{q^{*}}$ is positive-definite and radially unbounded. With $\tilde{x}$ partitioned as $\left[\begin{array}{ll}\tilde{x}_{\mathbb{E}}^{\prime} & \tilde{x}_{\mathbb{C}}^{\prime}\end{array}\right]^{\prime}$ it can therefore be concluded that $x_{\mathbb{E}}$ converges to $\tilde{x}_{\mathbb{E}}$ and $x_{\mathbb{C}}$ converges to $\tilde{x}_{\mathbb{C}}$ as $t \rightarrow \infty$ because of Eq. (25).
Corollary 1 guarantees that $y_{q^{*}}=0$ at $\tilde{x}$ or equivalently that $C_{q^{*}}\left(\tilde{x}_{\mathbb{E}}\right)=0$. In view of Eq. (22), $y=e_{q^{*}}+C_{q^{*}}\left(x_{\mathbb{E}}\right)$. Therefore $\lim _{t \rightarrow \infty} y=\lim _{t \rightarrow \infty} e_{q^{*}}$. Now $\lim _{t \rightarrow \infty} Y_{q^{*}}\left(e_{q^{*}}\right)=0$ because $(\mathrm{d} / \mathrm{d} t) Y_{q^{*}}\left(e_{q^{*}}\right)$ is bounded on $[0, \infty)$ and $Y_{q^{*}}\left(e_{q^{*}}\right) \in \mathscr{L}^{1}[0, \infty)$ (cf. [1], Lemma 1, p. 58). From this it follows that $\lim _{t \rightarrow \infty} y=\lim _{t \rightarrow \infty} e_{q^{*}}=0$ since $Y_{q^{*}}$ is positivedefinite and radially unbounded. We have proved the following.

Theorem 3. Let Assumptions 1-3 hold. For each initial state $x_{\mathbb{P}}(0) \in \mathscr{X}_{\mathbb{P}}, x_{\mathbb{E}}(0) \in \mathscr{X}_{\mathbb{E}}, x_{\mathbb{C}}(0) \in \mathscr{X}_{\mathbb{C}}, \pi_{p}(0)$ $>0, \quad p \in \mathscr{P}, \sigma(0) \in \mathscr{P}$, the solution $\left\{x_{\mathbb{P}}, x_{\mathbb{E}}, x_{\mathbb{C}}, \pi_{1}\right.$, $\left.\pi_{2}, \ldots, \pi_{m}\right\}$ to Eqs. (21)-(23) and (26) \{when $\sigma$ is the output of $\left.\mathbb{S}_{\mathbb{A}}\right\}$ exists and is bounded on $[0, \infty)$. Moreover, $y$ converges to zero as $t \rightarrow \infty$.

\section{Concluding remarks}

Throughout this paper we have made use of "linear" estimation errors of the form $e_{p}=y_{p}-y$. Given the nonlinear nature of the processes under consideration, it is natural to ask if the results in this paper would still hold if one were to utilize more general "nonlinear" errors. Towards this end, let us agree to call a locally Lipschitz continuous binary operation $\vee: \mathscr{Y} \times \mathscr{Y} \rightarrow \mathscr{Y}$, a generalized sum if, for all $y, \bar{y} \in \mathscr{Y}, y \vee \bar{y}=0 \Leftrightarrow y+\bar{y}=0$ and there is a locally Lipschitz continuous binary operation $\wedge: \mathscr{Y} \times \mathscr{Y} \rightarrow \mathscr{Y}$ such that $y=-(-y \wedge \bar{y}) \vee \bar{y}$ for all $y, \bar{y} \in \mathscr{Y}$. For example, if $\vee$ were defined so that $y \vee \bar{y} \triangleq y^{3}+\bar{y}^{3}$, then the definition of $\wedge$ would have to be $y \wedge \bar{y} \triangleq\left(y+\bar{y}^{3}\right)^{1 / 3}$. If $\vee$ were an associative operation, then $\wedge$ and $\vee$ would be the same.

Now, consider a supervisory control system in which for $p \in \mathscr{P}, e_{p}$ is of the form $e_{p} \triangleq-y \vee y_{p}$, where $\vee$ is a suitably defined generalized sum, possibly depending on $p$. Suppose that $\wedge$ is a corresponding binary operation such that $y=-(-y \wedge \bar{y}) \vee \bar{y}$ for all $y, \bar{y} \in \mathscr{Y}$. It is a simple matter to prove that if the $e_{p}$ are so defined, and if the signals coming out of the summing junctions in Figs. 3 and 4 are interpreted as $-v \wedge \bar{y}_{p}$ and $-y \vee y_{p}$, respectively, then Theorems 1 and 2 as well as Corollary 1 will still be true.

The main objective of this paper has been to make precise and justify a statement applicable to many adaptive algorithms, namely that certainty equivalence implies detectability. The intent of Section 9 has been to illustrate how this implication might be used in analyzing an adaptive system. Theorem 1 is very general. 
It applies to off-the-shelf, nonadaptive controllers such as back-stepping designed controllers [6,7], as well as to those specifically crafted to adaptive applications. The theorem's validity does not require the process model to be exactly matched by one of the nominal models in $\mathscr{M}$. In fact, all Theorem 1 requires of the process model is that it be detectable about each of its equilibrium input-output pairs.

\section{Appendix}

Again let $\dot{x}=A(x, u), \quad y=C(x, u)$ be a finitedimensional dynamical system whose state, input and output take values in real, finite-dimensional spaces $\mathscr{X}, \mathscr{U}$ and $\mathscr{Y}$, respectively - and suppose that $A$ and $C$ are at least locally Lipschitz continuous on $\mathscr{X} \oplus \mathscr{U}$. To prove Theorem 1 we use the following generalizations of concepts and results from [13].

By a supply pair for $A$ on a nonempty subset $\mathscr{S} \subset \mathscr{X} \oplus \mathscr{U}$ about an equilibrium input $\tilde{u}$, is meant any ordered pair of scalar-valued, positive-definite, continuous, unbounded, strictly increasing functions $\mathfrak{X}$ and $\mathfrak{U}$ on $[0, \infty)$, for which there is a continuously differentiable, positive-definite, radially unbounded \{storage\} function $V$ such that

$$
\begin{gathered}
\frac{\partial V(x-\tilde{x})}{\partial x} A(x, u) \leqslant- \\
\forall\{x(\|x-\tilde{x}\|)+\mathfrak{U}(\|u-\tilde{u}\|), \\
\forall\{\} \in \mathscr{S} .
\end{gathered}
$$

Here $\tilde{x}$ is any state such that $A(\tilde{x}, \tilde{u})=0$. By a supply triple for the pair $(C, A)$ about an equilibrium input-output pair $\{\tilde{u}, \tilde{y}\}$ is meant any ordered triple of scalar-valued, positive-definite, continuous, unbounded, strictly increasing functions $\mathfrak{X}, \mathfrak{U}$, and $\mathfrak{Y}$ on $[0, \infty)$, for which there is a continuously differentiable, positive-definite, radially unbounded \{storage function $V$ such that

$$
\begin{aligned}
& \frac{\partial V(x-\tilde{x})}{\partial x} A(x, u) \leqslant-\mathfrak{X}(\|x-\tilde{x}\|)+\mathfrak{U}(\|u-\tilde{u}\|) \\
& +\mathfrak{Y}(\|C(x, u)-\tilde{y}\|), \quad \forall\{x, u\} \in \mathscr{X} \oplus \mathscr{U} .
\end{aligned}
$$

Here $\tilde{x}$ is any vector such that $A(\tilde{x}, \tilde{u})=0$ and $C(\tilde{x}, \tilde{u})=\tilde{y}$. Like supply pairs, supply triples can also be defined on subsets $\mathscr{S} \subset \mathscr{X} \oplus \mathscr{U}$.

Suppose that $\tilde{x} \in \mathscr{X}, \tilde{u} \in \mathscr{U}$ and $\tilde{y} \in \mathscr{Y}$ are vectors such that $A(\tilde{x}, \tilde{u})=0$ and $C(\tilde{x}, \tilde{u})=\tilde{y}$. Then it is easy to see that if $\{\mathfrak{X}, \mathfrak{U}\}$ is a supply pair for $A$ on $\mathscr{X} \oplus \mathscr{U}$ about $\tilde{x}$, and $V$ is a corresponding storage function, then $\{V, \mathfrak{X}(\|\cdot\|), \mathfrak{U}(\|\cdot\|)\}$ is a stability triple for $A$ about $\tilde{u}$. Conversely, if $\{V, X, U\}$ is a stability triple for $A$ about $\tilde{u}$, and we define

$\mathfrak{X}(r) \triangleq \frac{r}{r+1} \inf _{\|x\| \geqslant r} X(x)$

and

$\mathfrak{U}(r) \triangleq r+\sup _{\|u\| \leqslant r} U(u)$

for $r \geqslant 0$, then $\{\mathfrak{X}, \mathfrak{U}\}$ is a supply pair for $A$ on $\mathscr{X} \oplus \mathscr{U}$ about $\tilde{u}$. In other words, $A$ is input-to-state stable about $\tilde{u}$ just in case it possesses a supply pair on $\mathscr{X} \oplus \mathscr{U}$ about $\tilde{u}$. By similar reasoning, $(C, A)$ is detectable about $\{\tilde{u}, \tilde{y}\}$ if and only if $(C, A)$ possesses a supply triple about $\{\tilde{u}, \tilde{y}\}$. The following result, proved in [13] for the case $\mathscr{S}=\mathscr{U} \oplus \mathscr{X}, \overline{\mathscr{S}}=\overline{\mathscr{U}} \oplus \overline{\mathscr{X}}$ enables one to establish the detectability the cascade connection of an input-to-state stable system with a detectable system.

Lemma A.1. Let $A: \mathscr{X} \oplus \mathscr{U} \rightarrow \mathscr{U}$ and $\bar{A}: \overline{\mathscr{X}} \oplus \overline{\mathscr{U}} \rightarrow \overline{\mathscr{U}}$ be maps, respectively, possessing supply pairs on $\mathscr{S} \subset \mathscr{X} \oplus \mathscr{U}$ about $\tilde{u}$ and on $\overline{\mathscr{S}} \subset \overline{\mathscr{X}} \oplus \overline{\mathscr{U}}$ about $\tilde{\bar{u}}$. There exist functions $\mathfrak{X}, \mathfrak{U}$ and $\overline{\mathfrak{U}}$ such that $\{\mathfrak{X}, \mathfrak{U}\}$ is a supply pair on $\mathscr{S}$ about $\tilde{u}$ for the former and with $\overline{\mathfrak{X}}(r) \triangleq 2 \mathfrak{U}(3 r), r \geqslant 0,\{\overline{\mathfrak{X}}, \overline{\mathfrak{U}}\}$ is a supply pair on $\overline{\mathscr{S}}$ about $\tilde{\bar{u}}$ for the latter.

With Lemma A.1 in hand, it is a simple matter to prove the following.

Lemma A.2. Let $\Sigma_{1}$ and $\Sigma_{2}$ be dynamical systems defined by

$\dot{x}_{1}=A_{1}\left(x_{1}, u_{1}\right), \quad y_{1}=C_{1}\left(x_{1}, u_{1}\right)$

and

$\dot{x}_{2}=A_{2}\left(x_{2}, u_{2}\right), \quad y_{2}=C_{2}\left(x_{2}, u_{2}\right)$,

respectively. Suppose that $\Sigma_{1}$ is detectable about $\left\{\tilde{u}_{1}, \tilde{y}_{1}\right\}$, that $\Sigma_{2}$ is input-to-state stable about $\tilde{u}_{2}$, and that $\tilde{u}_{1}=C_{2}\left(\tilde{x}_{2}, \tilde{u}_{2}\right)$ where $\tilde{x}_{2}$ is the equilibrium state of $\Sigma_{2}$ induced by $\tilde{u}_{2}$. Then the cascade interconnection of $\Sigma_{1}$ and $\Sigma_{2}$ determined by setting $u_{1}=y_{2}$, is detectable about $\left\{\tilde{u}_{2}, \tilde{y}_{1}\right\}$.

A proof of this lemma can be found in [3].

Proof of Theorem 1. With $\tilde{e}_{p} \triangleq 0$, let $\left\{\tilde{w}, \tilde{e}_{p}\right\}$ be an equilibrium input-output pair of the system $\Sigma_{p}$ shown in Fig. 4 and defined by Eqs. (9)-(11). Let $\tilde{x}_{\mathbb{P}}, \tilde{x}_{\mathbb{E}}, \tilde{x}_{\mathbb{C}}, \tilde{u}, \tilde{y}$ and $\tilde{y}_{p}$ be such that $A_{\mathbb{P}}\left(\tilde{x}_{\mathbb{P}}, \tilde{w}, \tilde{u}\right)=0$, 
$A_{\mathbb{E}}\left(\tilde{x}_{\mathbb{E}}, \tilde{u}, \tilde{y}\right)=0, \quad F_{p}\left(\tilde{x}_{\mathbb{C}}, \tilde{y}\right)=0, \quad \tilde{y}=C_{\mathbb{P}}\left(\tilde{x}_{\mathbb{P}}, \tilde{w}\right), \quad \tilde{u}=$ $G_{p}\left(\tilde{x}_{\mathbb{C}}, \tilde{y}\right)$, and $\tilde{y}_{p}=C_{p}\left(\tilde{x}_{\mathbb{E}}\right)$. Then $\{\{\tilde{w}, \tilde{u}\}, \tilde{y}\}$ is an equilibrium input-output pair of $\mathbb{P}$. By assumption, $\mathbb{P}$ is detectable about each such pair. Thus there must be a detectability quadruple $\{V, X, Y, U\}$ for $\mathbb{P}$ about $\{\{\tilde{w}, \tilde{u}\}, \tilde{y}\}$.

Let $\Sigma_{1}$ denote the system consisting of the dynamics of $\mathbb{P}$ given by Eq. (10) and the output estimation error $e_{p}$ defined by Eq. (9), i.e., the system

$\dot{x}_{\mathbb{P}}=A_{\mathbb{P}}\left(x_{\mathbb{P}}, w, u\right), \quad e_{p}=y_{p}-C_{\mathbb{P}}\left(x_{\mathbb{P}}, w\right)$.

In the sequel, we shall regard $\Sigma_{1}$ as a dynamical system with input $\left\{w, u, y_{p}\right\}$ and output $e_{p}$. Since $\tilde{e}_{p}=0$ and consequently $\tilde{y}_{p}=\tilde{y}$, it must be true that $\{\{\tilde{w}, \tilde{u}, \tilde{y}\}, 0\}$ is an equilibrium input-output pair of $\Sigma_{1}$. Moreover if

$$
\mathfrak{Y}(r) \triangleq \sup _{\|y\| \leqslant r} Y(\|y\|), \quad r \geqslant 0, \quad y \in \mathscr{Y},
$$

$\bar{U}\left(w, u, y_{p}\right) \triangleq U(w, u)+\mathfrak{Y}\left(2\left\|y_{p}\right\|\right)$, and $\bar{Y}\left(e_{p}\right) \triangleq$ $\mathfrak{Y}\left(2\left\|e_{p}\right\|\right)$, then $\{V, X, \bar{U}, \bar{Y}\}$ will be a detectability quadruple for $\Sigma_{1}$ about $\{\{\tilde{w}, \tilde{u}, \tilde{y}\}, 0\}$. Therefore $\Sigma_{1}$ is detectable about this pair.

Using the definition of $A_{p q}$ in Eq. (12) together with the definition of $e_{p}$ in Eq. (9) it is possible to write the estimator-controller subsystem defined by Eq. (11) as

$\dot{x}=A_{p p}(x, v)$,

where $v=e_{p}$ and $x \triangleq\left[x_{\mathbb{E}}^{\prime}, x_{C}^{\prime}\right]^{\prime}$. In the sequel it will be convenient to regard Eq. (A.4) as a dynamical system $\Sigma_{2}$ with input $\{w, v\}$ and output $\left\{w, u, y_{p}\right\}$ where $y_{p}=C_{p}\left(x_{\mathbb{E}}\right)$; the readout formula for $u$, namely $u=G_{p}\left(x_{\mathbb{C}}, C_{p}\left(x_{\mathbb{E}}\right)-v\right)$ is obtained from Eq. (11) by substituting $C_{p}\left(x_{\mathbb{E}}\right)-e_{p}$ for $y$. As noted in the discussion just preceding Theorem $2, A_{p p}(x, v)$ is inputto-state stable about the equilibrium input $\tilde{v}=0$. Thus $\Sigma_{2}$ is input-to-state stable about $\{\tilde{w}, 0\}$.

Let $\Sigma$ denote the system with input $\{w, v\}$ and output $e_{p}$ which is defined by the cascade connection of $\Sigma_{1}$ and $\Sigma_{2}$. Since $\Sigma_{1}$ is detectable about $\{\{\tilde{w}, \tilde{u}, \tilde{y}\}, 0\}$ and $\Sigma_{2}$ is input-to-state stable about $\{\tilde{w}, 0\}$, we can conclude, by Lemma A.2, that $\Sigma$ is detectable about $\{\{\tilde{w}, 0\}, 0\}$. In view of the definition of detectability, it is clear that this will remain true even if $v$ is set equal to $e_{p}$.

\section{References}

[1] M.A. Aizerman, F.R. Gantmacher, Absolute Stability of Regulator Systems, Holden-Day, San Franscisco, CA, 1964.

[2] E.D. Sontag, Smooth stabilization implies coprime factorization, IEEE Trans. Automat. Control 34(4) (1989) 435443.

[3] J.P. Hespanha, Logic-based switching algorithms in control, Ph.D. Thesis, Yale University, 1998.

[4] J.P. Hespanha, A.S. Morse, Certainty equivalence implies detectability, Proc. NOLCOS, 1998.

[5] J.P. Hespanha, A.S. Morse, Scale-independent hysteresis switching, Technical Report, Yale University, May 1998.

[6] I. Kanellakopoulos, P.V. Kokotovic, A.S. Morse, A toolkit for nonlinear feedback design, Systems Control Lett. 18 (1992) 83-92.

[7] M. Krstić, I. Kanellakopoulos, P. Kokotović, Nonlinear adaptive control design, in: Adaptive and Learning Systems for Signal Processing, Communications, and Control, Wiley, New York, 1995.

[8] A.S. Morse, Control using logic-based switching, in: A. Isidori (Ed.), Trends in Control, Springer, Berlin, 1995, pp. 69-113.

[9] A.S. Morse, Supervisory control of families of linear set-piont controllers - Part 1: exact matching, IEEE Trans. Automat. Control (1996) 1413-1431.

[10] A.S. Morse, Towards a unified theory of parameter adaptive control - Part 2: certainty equivalence and implicit tuning, IEEE Trans. Automat. Control 37(1) (1992) 15-29.

[11] A.S. Morse, F.M. Pait, Mimo design models and internal regulators for cyclicly-switched parameter-adaptive control systems, IEEE Trans. Automat. Control 39(9) (1994) 1809-1818.

[12] J.W. Polderman, I. Mareels, Adaptive Systems - An Introduction, Birkhäuser, Basel, 1996.

[13] E.D. Sontag, A. Teel, Changing supply functions in input/ state stable systems, IEEE Trans. Automat. Control 40(8) (1995) 1476-1478.

[14] E.D. Sontag, Y. Wang, Detectability of nonlinear systems, Proc. 30th CISS, vol. 2, 1996, pp. 1031-1036.

[15] E.D. Sontag, Y. Wang, New characterizations of input-tostate stability, IEEE Trans. Automat. Control 41(9) (1996) 1283-1294.

[16] E.D. Sontag, Y. Wang, On characterizations of the inputto-state stability property, Systems Control Lett. 24 (1995) 351-359.

[17] E.D. Sontag, Y. Wang, Output-to-state stability and detectability of nonlinear systems, Systems Control Lett. 29(5) (1997) 279-290. 